\title{
Hepatitis $B$ and $C$ virus infection among healthcare workers in Africa: a systematic review and meta-analysis
}

\author{
Daniel Atlaw ${ }^{1 *}$ D, Biniyam Sahiledengle ${ }^{2}$ and Zerihun Tariku ${ }^{3}$
}

\begin{abstract}
Background: Healthcare workers are at risk of acquiring hepatitis B and C virus infections through patients' blood and bodily fluids exposure. So far, there is no pooled data that shows the prevalence of HBV and HCV among health care workers in Africa. This study aimed to determine the pooled prevalence of hepatitis $B$ and $C$ infections among health care workers in Africa.

Methods: Studies reporting the prevalence of HBV and HCV were identified from major databases and gray literature. PubMed, CINAHL, POPLINE, ScienceDirect, African Journals Online (AJOL), and Google Scholar were systematically searched to identify relevant studies. A random-effect model was used to estimate the pooled prevalence of hepatitis B and C among health care workers in Africa. The heterogeneity of studies was assessed using Cochran $\mathrm{Q}$ statistics and $\mathrm{I}^{2}$ tests. Publication bias was assessed using Begg's tests.

Result: In total, 1885 articles were retrieved, and 44 studies met the inclusion criteria and included in the final analysis. A total of 17,510 healthcare workers were included. The pooled prevalence of hepatitis B virus infection among health care workers in Africa is estimated to be $6.81 \%$ (95\% Cl 5.67-7.95) with a significant level of heterogeneity $\left(I^{2}=91.6 \% ; p<0.001\right)$. While the pooled prevalence of hepatitis $C$ virus infection using the randomeffects model was 5.58\% (95\% Cl 3.55-7.61) with a significant level of heterogeneity $\left(I^{2}=95.1 \% ; p<0.001\right)$.

Conclusion: Overall, one in fifteen and more than one in twenty healthcare workers were infected by HBV and $\mathrm{HCV}$, respectively. The high burden of HBV and HCV infections remains a significant problem among healthcare workers in Africa.
\end{abstract}

Keywords: Hepatitis B, Hepatitis C, Health care workers, Africa

\section{Background}

Hepatitis B virus (HBV) is a DNA virus and hepatitis C virus ( $\mathrm{HCV}$ ) is an RNA virus [1]. Both $\mathrm{HBV}$ and $\mathrm{HCV}$ are transmitted by parenteral or mucosal exposure to infected blood and body fluids [1, 2]. Hepatitis B and C viruses are the most common causes of chronic hepatitis, cirrhosis, and hepatocellular carcinoma resulting in high

\footnotetext{
* Correspondence: danielatmwu@gmail.com

'Department of Human Anatomy, School of Medicine, Goba Referral Hospital, Madda Walabu University, Goba, Ethiopia

Full list of author information is available at the end of the article
}

morbidity and mortality all over the world $[3,4]$. Hepatitis B virus is more contagious [2] and HCV is a predominant cause of chronic hepatitis [5].

About 350 million people are chronically infected with HBV [6], and 150 million people have chronic hepatitis $\mathrm{C}$ virus infection [7]. According to the World Health Organization (WHO), about 14 million people are chronically infected with hepatitis $\mathrm{B}$, and nine million people are chronically infected with hepatitis $C$ in the European region in 2011 [8]. The majority of HBV and $\mathrm{HCV}$ infected cases are living in developing countries of

(c) The Author(s). 2021 Open Access This article is licensed under a Creative Commons Attribution 4.0 International License, which permits use, sharing, adaptation, distribution and reproduction in any medium or format, as long as you give appropriate credit to the original author(s) and the source, provide a link to the Creative Commons licence, and indicate if changes were made. The images or other third party material in this article are included in the article's Creative Commons licence, unless indicated otherwise in a credit line to the material. If material is not included in the article's Creative Commons licence and your intended use is not permitted by statutory regulation or exceeds the permitted use, you will need to obtain permission directly from the copyright holder. To view a copy of this licence, visit http://creativecommons.org/licenses/by/4.0/ The Creative Commons Public Domain Dedication waiver (http://creativecommons.org/publicdomain/zero/1.0/) applies to the data made available in this article, unless otherwise stated in a credit line to the data. 
sub-Saharan Africa [6]. Globally, HBV and HCV together accounted for an estimated 1.34 million deaths in the year 2015 [9] and in 2013 viral hepatitis infection was the seventh foremost cause of global mortality [10].

The transmission risk of viral hepatitis among health care workers (HCWs) is of great concern. The risk of acquiring hepatitis $\mathrm{B}$ virus by $\mathrm{HCWs}$ is four times greater than that of the general population [11, 12]. Healthcare workers are usually infected by HBV and HCV via occupational exposure to blood and bodily fluids [11, 13-15]. The circumstance is awful in Africa and Asia where 90\% of worldwide hepatitis infections occur [16, 17]. In developing countries, $40-60 \%$ of $\mathrm{HBV}$ infections in $\mathrm{HCW}$ were attributed to professional hazards [18]. Health care workers are vulnerable to contaminated sharp injuries which constitute a major source of hepatitis B infection, with an estimated 66,000 cases and 261 deaths annually in developing countries $[19,20]$. Further, about half of African HCWs are occupationally exposed to blood and body fluids [21, 22]. The evidence available suggests that many HCWs in Africa are at higher risk of hepatitis B infection [23].

Understanding the relative contribution of $\mathrm{HBV}$ and $\mathrm{HCV}$ to liver disease burden is important for setting public health priorities and guiding prevention programs [24]. In spite of recommendations on hepatitis B vaccination, the immunization rates among health professionals have remained consistently low in African countries [3]. A meta-analysis conducted in 2018 also reported that only a quarter of African HCWs were fully vaccinated against hepatitis $B$ virus [23].

So far, different studies have been conducted in Africa about the prevalence of $\mathrm{HBV}$ and $\mathrm{HCV}$ infections among HCWs, but the finding was inconsistent on HBV [2557] and HVC [58-73], and the pooled prevalence is still uncertain. For instance, the prevalence of HBV was $1.4 \%$ in Egypt [33], 2.4\% in Ethiopia [29], 17.8\% in Senegal [54], and $25.7 \%$ in Nigeria [55], while the prevalence of HCV was $0.4 \%$ in Ethiopia [60], 1.3 in Rwanda [65], and $16.7 \%$ in Egypt [58]. Up to date, no pooled data estimate shows the prevalence of $\mathrm{HBV}$ and $\mathrm{HCV}$ among HCWs in Africa. Therefore, this study aimed to determine the pooled prevalence of hepatitis $\mathrm{B}$ and $\mathrm{C}$ infections among HCWs in Africa.

\section{Methods}

\section{Study design and reporting}

The protocol of this study was registered in the International Prospective Register of Systematic Reviews (PROSPERO), the University of York Centre for Reviews and Dissemination (ID number CRD42021230905). A systematic review and meta-analysis were conducted to estimate the pooled prevalence of HBV and HCV among HCWs in Africa. This review and meta-analysis were conducted according to the guideline of Preferred Reporting Items for Systematic reviews and MetaAnalysis (PRISMA) (Supplementary file 1).

\section{Eligibility criteria}

Studies conducted in Africa that have reported the prevalence of hepatitis B or/and hepatitis $\mathrm{C}$ and fulfilled the following criteria were included.

- Population. Healthcare works (HCWs) with direct contact to patients

- Study designs. Observational studies reporting the prevalence of hepatitis B or/and hepatitis $C$ were eligible for this systematic review and meta-analysis

- Language. Articles published in English were considered.

- Publication status. Both published and unpublished articles were considered.

- Year of publication. All publications reported up to December 31, 2020, were considered.

- Exclusion criteria. Studies that reported hepatitis B prevalence but do not have a separate outcome for HBsAg

\section{Operational definition}

Healthcare workers are referred to as full-time employees working in a healthcare setting whose activities involve direct contact with patients. Hence, we incorporated studies, which have involved physicians, nurses, and laboratory technicians mainly.

\section{Outcome of interests and measurement}

Prevalence hepatitis $B$ and $C$ virus infection were the outcome of interests. The prevalence of HBV was calculated from primary studies by dividing the number of HCWs tested for HBsAg and reported as positive to the total number of health care workers multiplied by 100 . Similarly, the prevalence of hepatitis $\mathrm{C}$ virus infection was calculated by dividing the number of HCWs tested for serum HCV antibody, and reported positive to the total number of health care workers multiplied by 100 .

\section{Search strategy}

A systematic search of works of literature was conducted by the authors to identify all relevant primary studies. Both published and unpublished articles on the prevalence of hepatitis B virus and hepatitis $C$ virus infections in Africa were identified through a literature search. The databases used to search for studies were PubMed, Science Direct, CINAHL, Popline, Cochrane Library, and African Journals Online (AJOL) and gray literature was searched on Google and Google Scholar until December 31, 2020. The following key search terms and Medical Subject Headings $[\mathrm{MeSH}]$ were used "prevalence" OR 
"magnitude" AND "hepatitis B" OR "hepatitis C" AND "health care workers" OR "health professionals" AND "Africa $[\mathrm{MeSH}]$ " were used separately or in combination with the Boolean operator's terms "AND" and "OR" (Supplementary file 2). In addition, the reference lists of the retrieved studies were also scanned to access additional articles and screened against our eligibility criteria.

\section{Data Extraction}

In this review, all the searched articles were exported into the EndNote version X8 software, and subsequently, the duplicate articles were removed. Screening of retrieved articles titles, abstracts, and full-text quality were conducted independently by two review authors (DA and BS) based on the eligibility criteria. Any disagreement between the two review authors was resolved by consensus through discussion. Afterward, full-text articles were retrieved and appraised to approve eligibility. Finally, the screened articles were compiled together by the two investigators. Data were extracted using a data extraction format in Microsoft Office Excel software. The data extraction tool consists of the name of the author(s), year of publication, country and sub-region, study design, sample size, and prevalence of hepatitis B and hepatitis $\mathrm{C}$ (Table 1).

\section{Risk of bias assessment}

The qualities of the included studies were assessed and the risks for biases were refereed using the Joanna Briggs Institute (JBI) quality assessment tool for the prevalence studies [74]. Two reviewers (DA and BS) assess the quality of included studies independently and the discrepancy between the two review authors was resolved by reaching a consensus through discussion. The assessment tool consists of nine parameters: (1) appropriate sampling frame, (2) proper sampling technique, (3) adequate sample size, (4) study subject and setting description, (5) sufficient data analysis, (6) use of valid methods for the identified conditions, (7) valid measurement for all participants, (8) using appropriate statistical analysis, and (9) adequate response rate [74]. Failure to satisfy each parameter was scored as 1 if not 0 . When the information provided was not adequate to assist in making a judgment for a specific item, we agreed to grade that item as 1 (failure to satisfy a specific item). The risks for biases were classified as either low (total score, 0 to 2), moderate (total score, 3 or 4), or high (total score, 5 to 9) (Table 2).

\section{Statistical methods and analysis}

The extracted data were imported into STATA version 14 software for statistical analysis. The heterogeneity among all included studies was assessed by $\mathrm{I}^{2}$ statistics and Cochran $\mathrm{Q}$ test. In this meta-analysis, the tests indicate that the presence of significant heterogeneity among included studies $\left(\mathrm{I}^{2}=91.6, P\right.$-value $\left.<0.001\right)$. Thus, a random-effects model was used to analyze the data. Pooled prevalence along their corresponding 95\% $\mathrm{CI}$ was presented using a forest plot. Sub-group analyses for the prevalence of hepatitis $B$ and $C$ were performed by sub-regions of Africa, sample size, year of publication, and professions of HCWs.

\section{Publication bias}

In this meta-analysis, the presence of publication bias was evaluated using funnel plots and Begg's tests at a significance level of less than 0.05 .

\section{Sensitivity analysis}

To identify the source of heterogeneity, a leave-one-out sensitivity analysis was employed.

\section{Results}

\section{Description of included studies}

About 1885 studies were retrieved from initial electronic searches using international databases and google search. The database included PubMed $(n=63)$, ScienceDirect $(n=68)$, Hinari $(n=71)$, Google Scholar $(n=879)$, Cochrane Library $(n=4)$, AJOL $(n=260)$, CINAHL $(n$ $=9)$, POPLINE $(n=490)$, and the remaining $(n=41)$ studies were identified through manual search. Of these, 1332 duplicates were removed, the remaining 553 articles were screened by title and abstract, and 460 articles were excluded after reading their titles and abstracts. Ninety-three full-text articles remained and were further assessed for their eligibility. Finally, based on the predefined inclusion and exclusion criteria, a total of 44 articles were included in the meta-analysis and data were extracted for the final analysis (Fig. 1).

\section{Characteristics of the included studies}

Of 44 articles included in this review and meta-analyses, 5 were conducted in Ethiopia, 2 in Uganda, 2 in Tanzania, 3 in Sudan, 8 in Nigeria, 6 in Egypt, 5 in Cameron, 2 in Morocco, 2 in Sierra Leone, 2 in Rwanda, 1 in Kenya, 1 in Côte d'Ivoire, 2 in south Africa, 1 in Senegal, 1 in DR Congo, and 2 in Libya (Table 1).

A total number of $17,510 \mathrm{HCW}$ s participated in this study. The lowest sample size was reported from Egypt $(\mathrm{n}=69)$ and the highest was from Cameron $(n=1790)$. Among the included studies, 31 of them reported the prevalence of $\mathrm{HBV}, 3$ of them presented the prevalence of $\mathrm{HCV}$, and 10 studies reported the prevalence of both HBV and HCV (Table 1).

The latest article was published in 2020, and the earliest study was concluded in 1989. The prevalence of hepatitis B among African HCWs ranged from 1.4\% in Egypt to $25.7 \%$ 
Table 1 Characteristics of included studies in meta-analysis on prevalence of hepatitis B and C in Africa

\begin{tabular}{|c|c|c|c|c|c|c|c|c|c|}
\hline Author name & $\begin{array}{l}\text { Year of } \\
\text { publication }\end{array}$ & Country & $\begin{array}{l}\text { Study } \\
\text { design }\end{array}$ & $\begin{array}{l}\text { Sample } \\
\text { size }\end{array}$ & $\begin{array}{l}\text { Prevalence } \\
\text { of HBV }\end{array}$ & $\begin{array}{l}\text { Prevalence } \\
\text { of HCV }\end{array}$ & $\begin{array}{l}\text { Prevalence } \\
\text { of } \mathrm{HBV} \text { in } \\
\text { nurses }\end{array}$ & $\begin{array}{l}\text { Prevalence of HBV } \\
\text { in laboratory } \\
\text { technician }\end{array}$ & $\begin{array}{l}\text { Prevalence of } \\
\text { HBV in } \\
\text { physician }\end{array}$ \\
\hline $\begin{array}{l}\text { Desalegn et al. } \\
\text { [27] }\end{array}$ & 2013 & Ethiopia & $\begin{array}{l}\text { Cross- } \\
\text { sectional }\end{array}$ & 254 & 2.4 & & 4 & & 3.8 \\
\hline $\begin{array}{l}\text { Ziraba et al. } \\
\text { [53] }\end{array}$ & 2010 & Uganda & $\begin{array}{l}\text { Cross- } \\
\text { sectional }\end{array}$ & 370 & 8.1 & & 8.61 & 18.18 & 3.8 \\
\hline $\begin{array}{l}\text { Mueller et al. } \\
\text { [51] }\end{array}$ & 2016 & Tanzania & $\begin{array}{l}\text { Cross- } \\
\text { sectional }\end{array}$ & 598 & 7 & & & & \\
\hline Nail et al. [50] & 2008 & Sudan & $\begin{array}{l}\text { Cross- } \\
\text { sectional }\end{array}$ & 211 & 2.3 & & 3.1 & & \\
\hline $\begin{array}{l}\text { Abiola et al. } \\
\text { [25] }\end{array}$ & 2016 & Nigeria & $\begin{array}{l}\text { Cross- } \\
\text { sectional }\end{array}$ & 134 & 1.5 & & 1.15 & & 2.23 \\
\hline $\begin{array}{l}\text { Abdelwahab } \\
\text { et al. [58] }\end{array}$ & 2012 & Egypt & $\begin{array}{l}\text { Cross- } \\
\text { sectional }\end{array}$ & 842 & 1.5 & 16.7 & & & \\
\hline Braka et al. [28] & 2006 & Uganda & $\begin{array}{l}\text { Cross- } \\
\text { sectional }\end{array}$ & 311 & 9 & & 10.58 & 11.11 & 2.44 \\
\hline $\begin{array}{l}\text { Djeriri et al. } \\
\text { [31] }\end{array}$ & 2008 & Morocco & $\begin{array}{l}\text { Cross- } \\
\text { sectional }\end{array}$ & 285 & 5 & & 1.5 & & 1.85 \\
\hline $\begin{array}{l}\text { Ngekeng et al. } \\
\text { [48] }\end{array}$ & 2018 & Cameroon & $\begin{array}{l}\text { Cross- } \\
\text { sectional }\end{array}$ & 281 & 5 & & & & \\
\hline $\begin{array}{l}\text { Elmaghloub } \\
\text { et al. [33] }\end{array}$ & 2017 & Egypt & $\begin{array}{l}\text { Cross- } \\
\text { sectional }\end{array}$ & 564 & 1.4 & & & & \\
\hline $\begin{array}{l}\text { Elmukashfi } \\
\text { et al. [34] }\end{array}$ & 2012 & Sudan & $\begin{array}{l}\text { Cross- } \\
\text { sectional }\end{array}$ & 843 & 6 & & & & \\
\hline $\begin{array}{l}\text { Elduma and } \\
\text { Saeed [36] }\end{array}$ & 2006 & Sudan & $\begin{array}{l}\text { Cross- } \\
\text { sectional }\end{array}$ & 245 & 4.9 & & & & \\
\hline $\begin{array}{l}\text { Fritzsche et al. } \\
\text { [59] }\end{array}$ & 2015 & Cameroon & $\begin{array}{l}\text { Cross- } \\
\text { sectional }\end{array}$ & 237 & 6.3 & 1.7 & 7.29 & 2.7 & 6.25 \\
\hline $\begin{array}{l}\text { Gebremariam } \\
\text { et al. [38] }\end{array}$ & 2018 & Ethiopia & $\begin{array}{l}\text { Cross- } \\
\text { sectional }\end{array}$ & 332 & 4.52 & & 4.3 & 4.44 & 5 \\
\hline Hebo et al. [60] & 2019 & Ethiopia & $\begin{array}{l}\text { Cross- } \\
\text { sectional }\end{array}$ & 240 & 2.5 & 0.4 & & & \\
\hline $\begin{array}{l}\text { Mafopa et al. } \\
\text { [62] }\end{array}$ & 2019 & $\begin{array}{l}\text { Sierra } \\
\text { Leone }\end{array}$ & $\begin{array}{l}\text { Cross- } \\
\text { sectional }\end{array}$ & 81 & 4.9 & 2.5 & & & \\
\hline Alese et al. [26] & 2016 & Nigeria & $\begin{array}{l}\text { Cross- } \\
\text { sectional }\end{array}$ & 187 & 1.1 & & & & \\
\hline $\begin{array}{l}\text { Munier et al. } \\
\text { [63] }\end{array}$ & 2013 & Egypt & Cohort & 597 & 7.3 & 7.2 & & & \\
\hline $\begin{array}{l}\text { Kisangau et al. } \\
\text { [57] }\end{array}$ & 2018 & Kenya & $\begin{array}{l}\text { Cross- } \\
\text { sectional }\end{array}$ & 295 & 4.5 & & & & \\
\hline $\begin{array}{l}\text { Jean-Baptiste } \\
\text { et al. [61] }\end{array}$ & 2018 & Ivory cost & $\begin{array}{l}\text { Cross- } \\
\text { sectional }\end{array}$ & 632 & 8.4 & 1.4 & 19.48 & 28 & 38.14 \\
\hline Souly et al. [64] & 2016 & Moroccoo & $\begin{array}{l}\text { Cross- } \\
\text { sectional }\end{array}$ & 1189 & 3.2 & 1.3 & 4.03 & 3.45 & 2.7 \\
\hline Orji et al. [47] & 2020 & Nigeria & $\begin{array}{l}\text { Cross- } \\
\text { sectional }\end{array}$ & 236 & 2.1 & & & & \\
\hline $\begin{array}{l}\text { Yizengaw et al. } \\
\text { [43] }\end{array}$ & 2018 & Ethiopia & $\begin{array}{l}\text { Cross- } \\
\text { sectional }\end{array}$ & 268 & 2.6 & & 1.87 & 6.45 & \\
\hline $\begin{array}{l}\text { Ndako et al. } \\
\text { [49] }\end{array}$ & 2014 & Nigeria & $\begin{array}{l}\text { Cross- } \\
\text { sectional }\end{array}$ & 188 & 17 & & 13.4 & 12.9 & 21.43 \\
\hline $\begin{array}{l}\text { Elikwu et al. } \\
\text { [32] }\end{array}$ & 2016 & Nigeria & $\begin{array}{l}\text { Cross- } \\
\text { sectional }\end{array}$ & 100 & 7 & & 7.02 & & 5.88 \\
\hline $\begin{array}{l}\text { Geberemicheal } \\
\text { et al. [37] }\end{array}$ & 2013 & Ethiopia & $\begin{array}{l}\text { Cross- } \\
\text { sectional }\end{array}$ & 110 & 7.3 & & & & \\
\hline
\end{tabular}


Table 1 Characteristics of included studies in meta-analysis on prevalence of hepatitis B and C in Africa (Continued)

\begin{tabular}{|c|c|c|c|c|c|c|c|c|c|}
\hline Author name & $\begin{array}{l}\text { Year of } \\
\text { publication }\end{array}$ & Country & $\begin{array}{l}\text { Study } \\
\text { design }\end{array}$ & $\begin{array}{l}\text { Sample } \\
\text { size }\end{array}$ & $\begin{array}{l}\text { Prevalence } \\
\text { of HBV }\end{array}$ & $\begin{array}{l}\text { Prevalence } \\
\text { of HCV }\end{array}$ & $\begin{array}{l}\text { Prevalence } \\
\text { of HBV in } \\
\text { nurses }\end{array}$ & $\begin{array}{l}\text { Prevalence of HBV } \\
\text { in laboratory } \\
\text { technician }\end{array}$ & $\begin{array}{l}\text { Prevalence of } \\
\text { HBV in } \\
\text { physician }\end{array}$ \\
\hline Shao et al. [46] & 2018 & Tanzania & $\begin{array}{l}\text { Cross- } \\
\text { sectional }\end{array}$ & 442 & 5.7 & & 3.7 & 10.81 & 5.38 \\
\hline $\begin{array}{l}\text { Sondlane et al. } \\
\text { [45] }\end{array}$ & 2016 & $\begin{array}{l}\text { South } \\
\text { Africa }\end{array}$ & $\begin{array}{l}\text { Cross- } \\
\text { sectional }\end{array}$ & 314 & 2.9 & & & & \\
\hline $\begin{array}{l}\text { Tatsilong et al. } \\
\text { [44] }\end{array}$ & 2016 & Cameroon & $\begin{array}{l}\text { Cross- } \\
\text { sectional }\end{array}$ & 100 & 11 & & 10.2 & 25 & \\
\hline $\begin{array}{l}\text { Kateera et al. } \\
\text { [65] }\end{array}$ & 2014 & Rwanda & $\begin{array}{l}\text { Cross- } \\
\text { sectional }\end{array}$ & 378 & 2.9 & 1.3 & & & \\
\hline $\begin{array}{l}\text { Elbahrawy et al. } \\
\text { [66] }\end{array}$ & 2017 & Egypt & $\begin{array}{l}\text { Cross- } \\
\text { sectional }\end{array}$ & 564 & & 8.7 & & & \\
\hline $\begin{array}{l}\text { Akazong et al. } \\
\text { [27] }\end{array}$ & 2020 & Cameroon & $\begin{array}{l}\text { Cross- } \\
\text { sectional }\end{array}$ & 338 & 10.6 & & 12.5 & 8.89 & 5.88 \\
\hline $\begin{array}{l}\text { Amiwero et al. } \\
\text { [67] }\end{array}$ & 2017 & Nigeria & $\begin{array}{l}\text { Cross- } \\
\text { sectional }\end{array}$ & 248 & 11.3 & 2.4 & 13.04 & & 11.76 \\
\hline Daw et al. [30] & 2000 & Libya & $\begin{array}{l}\text { Cross- } \\
\text { sectional }\end{array}$ & 459 & 4 & & & & \\
\hline $\begin{array}{l}\text { Romieu et al. } \\
\text { [54] }\end{array}$ & 1989 & Senegal & $\begin{array}{l}\text { Cross- } \\
\text { sectional }\end{array}$ & 775 & 17.8 & & & & \\
\hline Qin et al. [52] & 2018 & $\begin{array}{l}\text { Sierra } \\
\text { Leone }\end{array}$ & $\begin{array}{l}\text { Cross- } \\
\text { sectional }\end{array}$ & 211 & 10 & & & & \\
\hline $\begin{array}{l}\text { Elzouki et al. } \\
\text { [35] }\end{array}$ & 2014 & Libya & Cohort & 601 & 1.8 & & 2.41 & 0.91 & 0.74 \\
\hline $\begin{array}{l}\text { Ndongo et al. } \\
{[56]}\end{array}$ & 2016 & Cameroon & $\begin{array}{l}\text { Cross- } \\
\text { sectional }\end{array}$ & 1790 & 8.7 & & & & \\
\hline $\begin{array}{l}\text { Vardas et al. } \\
\text { [68] }\end{array}$ & 2002 & $\begin{array}{l}\text { South } \\
\text { Africa }\end{array}$ & $\begin{array}{l}\text { Cross- } \\
\text { sectional }\end{array}$ & 399 & & 1.8 & & & \\
\hline $\begin{array}{l}\text { Lungosi et al. } \\
{[41]}\end{array}$ & 2018 & DR Congo & $\begin{array}{l}\text { Cross- } \\
\text { sectional }\end{array}$ & 97 & 18.6 & & & & \\
\hline $\begin{array}{l}\text { Massaquoi } \\
\text { et al. [39] }\end{array}$ & 2018 & $\begin{array}{l}\text { Sierra } \\
\text { Leone }\end{array}$ & $\begin{array}{l}\text { Cross- } \\
\text { sectional }\end{array}$ & 447 & 8.7 & & & & \\
\hline $\begin{array}{l}\text { Mbaawuaga } \\
\text { et al. [40] }\end{array}$ & 2019 & Nigeria & $\begin{array}{l}\text { Cross- } \\
\text { sectional }\end{array}$ & 221 & 10.6 & & 11.63 & & \\
\hline Sani et al. [69] & 2011 & Nigeria & $\begin{array}{l}\text { Cross- } \\
\text { sectional }\end{array}$ & 100 & 19 & 5 & & & \\
\hline Zayet et al. [72] & 2015 & Egypt & $\begin{array}{l}\text { Cross- } \\
\text { sectional }\end{array}$ & 215 & 3.1 & 5.2 & 32 & & 14.29 \\
\hline $\begin{array}{l}\text { Kefenie et al. } \\
\text { [42] }\end{array}$ & 1989 & Ethiopia & $\begin{array}{l}\text { Cross- } \\
\text { sectional }\end{array}$ & 432 & 9.2 & & 8.82 & 6.45 & \\
\hline $\begin{array}{l}\text { El-Sokkary et al. } \\
\text { [70] }\end{array}$ & 2017 & Egypt & $\begin{array}{l}\text { Cross- } \\
\text { sectional }\end{array}$ & 69 & & 40.6 & & & \\
\hline Belo et al. [55] & 2000 & Nigeria & $\begin{array}{l}\text { Cross- } \\
\text { sectional }\end{array}$ & 167 & 25.7 & & & & \\
\hline $\begin{array}{l}\text { Gyang et al. } \\
\text { [73] }\end{array}$ & 2016 & Nigeria & $\begin{array}{l}\text { Cross- } \\
\text { sectional }\end{array}$ & 155 & 8.5 & 6.5 & 2.74 & & 3.13 \\
\hline
\end{tabular}

in Nigeria. The prevalence of hepatitis $C$ varied from $0.4 \%$ in Ethiopia to $40.6 \%$ in Egypt (Table 1).

\section{Socioeconomic status of African countries included in this meta-analysis}

Out of sixteen countries included in this meta-analysis, $6(37.50 \%)$ of them have gross national income (GNI) per capita less than $\$ 1036,8$ (50\%) of them have GNI per capita between $\$ 1036$ to 4036 , and 2 (12.50\%) of them have GNI per capita above $\$ 4036$. In terms of governmental health care expenditure, about 9 (56.25\%) of included countries have less than $30 \%$ health expenditure from domestic government funding and 2 (12.50\%) have higher than $50 \%$ of health expenditure from 
Table 2 Risk bias assessment of individual studies included for meta-analysis on prevalence of hepatitis B and C in Africa

\begin{tabular}{|c|c|c|c|c|c|c|c|c|c|c|c|c|}
\hline Wow & Year of publication & Q1 & Q2 & Q3 & Q4 & Q5 & Q6 & Q7 & Q8 & Q9 & Total score & Risk of bias \\
\hline Desalegn et al. [29] & 2013 & 1 & 0 & 0 & 0 & 0 & 1 & 1 & 0 & 0 & 3 & Moderate \\
\hline Ziraba et al. [53] & 2010 & 0 & 0 & 0 & 0 & 0 & 0 & 0 & 0 & 0 & 0 & Low \\
\hline Mueller et al. [51] & 2016 & 0 & 0 & 0 & 0 & 0 & 1 & 0 & 0 & 0 & 1 & Low \\
\hline Nail et al. [50] & 2008 & 1 & 1 & 0 & 1 & 0 & 0 & 0 & 1 & 1 & 5 & High \\
\hline Abdelwahab et al. [58] & 2012 & 1 & 0 & 1 & 0 & 0 & 1 & 1 & 1 & 0 & 5 & High \\
\hline Braka et al. [28] & 2006 & 0 & 1 & 0 & 0 & 0 & 0 & 0 & 1 & 0 & 2 & Low \\
\hline Djeriri et al. [31] & 2008 & 1 & 0 & 0 & 0 & 0 & 0 & 0 & 0 & 1 & 2 & Low \\
\hline Ngekeng et al. [48] & 2018 & 0 & 0 & 0 & 0 & 1 & 1 & 0 & 1 & 1 & 4 & Moderate \\
\hline Elmaghloub et al. [33] & 2017 & 1 & 0 & 0 & 1 & 0 & 0 & 0 & 0 & 1 & 3 & Moderate \\
\hline Elmukashfi et al. [34] & 2012 & 0 & 1 & 0 & 1 & 0 & 0 & 0 & 1 & 1 & 4 & Moderate \\
\hline Elduma and Saeed [36] & 2006 & 1 & 0 & 0 & 1 & 0 & 0 & 0 & 1 & 1 & 4 & Moderate \\
\hline Fritzsche et al. [59] & 2015 & 1 & 0 & 0 & 1 & 0 & 1 & 1 & 0 & 1 & 5 & High \\
\hline Gebremariam et al. [38] & 2018 & 0 & 0 & 0 & 0 & 0 & 1 & 0 & 0 & 0 & 1 & Low \\
\hline Munier et al. [63] & 2013 & 1 & 0 & 0 & 1 & 0 & 0 & 0 & 0 & 1 & 3 & Moderate \\
\hline Kisangau et al. [57] & 2018 & 0 & 0 & 0 & 0 & 1 & 0 & 0 & 1 & 0 & 2 & Low \\
\hline Jean-Baptiste et al. [61] & 2018 & 1 & 0 & 0 & 1 & 0 & 1 & 0 & 0 & 1 & 4 & Moderate \\
\hline Souly et al. [64] & 2016 & 1 & 0 & 0 & 1 & 0 & 0 & 0 & 0 & 1 & 3 & Moderate \\
\hline Orji et al. [47] & 2020 & 0 & 0 & 0 & 0 & 0 & 0 & 0 & 1 & 0 & 1 & Low \\
\hline Yizengaw et al. [43] & 2018 & 0 & 0 & 0 & 0 & 0 & 0 & 0 & 0 & 0 & 0 & Low \\
\hline Ndako et al. [49] & 2014 & 0 & 0 & 0 & 1 & 0 & 1 & 1 & 0 & 1 & 4 & Moderate \\
\hline Elikwu et al. [32] & 2016 & 1 & 0 & 0 & 1 & 0 & 0 & 0 & 1 & 0 & 3 & Moderate \\
\hline Geberemicheal et al. [37] & 2013 & 1 & 0 & 0 & 1 & 0 & 0 & 0 & 0 & 0 & 2 & Low \\
\hline Shao et al. [46] & 2018 & 0 & 0 & 0 & 0 & 1 & 1 & 0 & 0 & 0 & 2 & Low \\
\hline Sondlane et al. [45] & 2016 & 0 & 0 & 0 & 0 & 0 & 0 & 1 & 0 & 0 & 1 & Low \\
\hline Tatsilong et al. [44] & 2016 & 0 & 0 & 0 & 0 & 0 & 1 & 0 & 1 & 0 & 2 & Low \\
\hline Kateera et al. [65] & 2014 & 0 & 0 & 0 & 0 & 1 & 0 & 0 & 1 & 0 & 2 & Low \\
\hline Elbahrawy et al. [66] & 2017 & 1 & 0 & 0 & 1 & 0 & 0 & 0 & 0 & 0 & 2 & Low \\
\hline Akazong et al. [27] & 2020 & 0 & 0 & 0 & 0 & 0 & 0 & 1 & 0 & 0 & 1 & Low \\
\hline Amiwero et al. [67] & 2017 & 0 & 0 & 1 & 0 & 0 & 0 & 1 & 1 & 0 & 3 & Low \\
\hline Daw et al. [30] & 2000 & 0 & 0 & 1 & 0 & 1 & 0 & 1 & 1 & 0 & 4 & Moderate \\
\hline Romieu et al. [54] & 1989 & 1 & 0 & 0 & 1 & 0 & 1 & 1 & 1 & 1 & 6 & High \\
\hline Qin et al. [52] & 2018 & 0 & 0 & 0 & 0 & 0 & 1 & 0 & 0 & 0 & 1 & Low \\
\hline Elzouki et al. [35] & 2014 & 0 & 0 & 0 & 0 & 1 & 0 & 0 & 0 & 0 & 1 & Low \\
\hline Ndongo et al. [56] & 2016 & 0 & 1 & 0 & 0 & 0 & 0 & 0 & 0 & 0 & 1 & Low \\
\hline Vardas et al. [68] & 2002 & 0 & 1 & 0 & 0 & 1 & 1 & 1 & 0 & 0 & 4 & Moderate \\
\hline Lungosi et al. [41] & 2018 & 0 & 0 & 0 & 0 & 1 & 0 & 1 & 0 & 0 & 2 & Low \\
\hline Massaquoi et al. [39] & 2018 & 0 & 0 & 0 & 0 & 0 & 0 & 0 & 1 & 0 & 1 & Low \\
\hline Mbaawuaga et al. [40] & 2019 & 1 & 0 & 0 & 0 & 0 & 0 & 0 & 1 & 0 & 2 & Low \\
\hline Sani et al. [69] & 2011 & 1 & 1 & 1 & 0 & 1 & 0 & 0 & 1 & 0 & 5 & High \\
\hline Zayet et al. [72] & 2015 & 0 & 1 & 1 & 0 & 1 & 0 & 1 & 1 & 1 & 6 & High \\
\hline Kefenie et al. [42] & 1989 & 1 & 0 & 0 & 1 & 0 & 0 & 0 & 0 & 0 & 2 & Low \\
\hline El-Sokkary et al. [70] & 2017 & 0 & 0 & 0 & 0 & 0 & 1 & 0 & 0 & 0 & 1 & Low \\
\hline Belo et al. [55] & 2000 & 1 & 1 & 1 & 0 & 0 & 0 & 0 & 0 & 1 & 4 & Moderate \\
\hline Gyang et al. [73] & 2017 & 0 & 0 & 0 & 1 & 0 & 0 & 0 & 0 & 0 & 1 & Low \\
\hline
\end{tabular}


The risk of bias was classified as either low (total score, 0 to 2), moderate (total score, 3 or 4), or high (total score, 5 to 9)

$\mathrm{Q} 1$ = Was the sample frame appropriate to address the target population?

Q2 = Were study participants sampled in an appropriate way?

Q3 = Was the sample size adequate?

Q4 = Were the study subjects and the setting described in detail?

Q5 = Was the data analysis conducted with sufficient coverage of the identified sample?

Q6 = Were valid methods used for the identification of the condition?

Q7 = Was the condition measured in a standard, reliable way for all participants?

$\mathrm{Q} 8=$ Was there appropriate statistical analysis?

Q9 = Was the response rate adequate, and if not, was the low response rate managed appropriately?

domestic government funding. In addition, out of the sixteen countries, 7 (43.75\%) of them have universal health coverage less than $50 \%$ and one country has universal health coverage greater than $60 \%$ (Table 3).

\section{Prevalence of hepatitis B and C infection among health} HCWs in Africa

The pooled prevalence of hepatitis B among HCWs in Africa using the random-effect model was estimated to be $6.81 \%$ (95\% CI 5.67-7.92) with a significant level of heterogeneity $\left(\mathrm{I}^{2}=91.6 \% ; p<0.001\right)$ (Fig. 2). While the pooled prevalence of hepatitis $\mathrm{C}$ using the randomeffects model was 5.58\% (95\% CI 3.55-7.61) with a significant level of heterogeneity $\left(\mathrm{I}^{2}=95.1 \% ; p<0.001\right)$ (Fig. 3).

\section{Sub-group analysis}

To identify the possible source of heterogeneity, subgroup analysis was conducted by sub-regions of Africa,

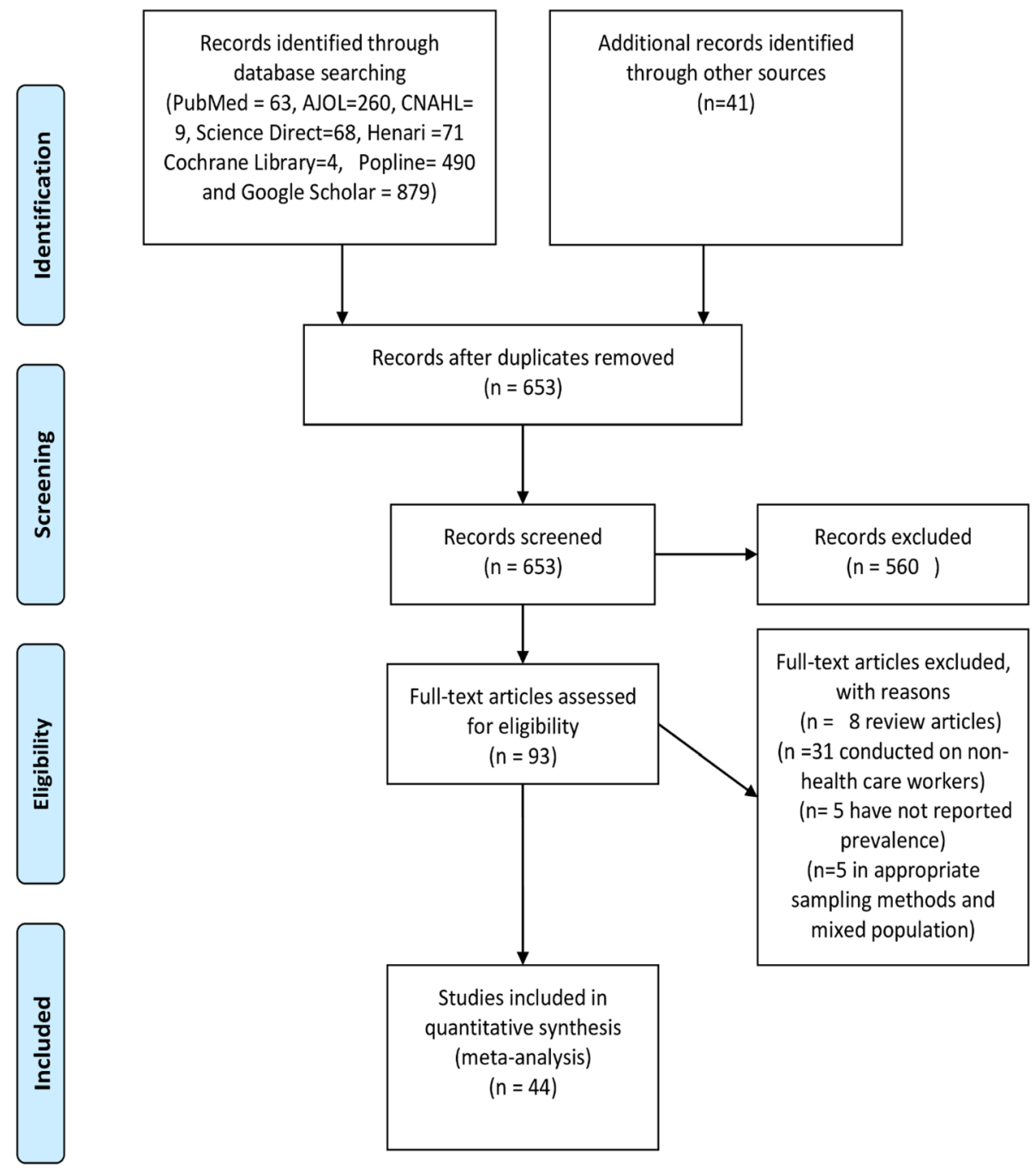

Fig. 1 Flow diagram of systemic review and meta-analysis on prevalence of hepatitis B and C among health care workers in Africa, 1989-2021 
Table 3 Socioeconomic characteristics of African countries included in meta-analysis for prevalence of HBV and HCV in Africa

\begin{tabular}{|c|c|c|c|c|}
\hline Countries & $\begin{array}{l}\text { GNI per capita (US\$) } \\
\text { (word bank.org 2019) }\end{array}$ & $\begin{array}{l}\text { Governmental health care expenditure } \\
\text { (\%) (africanhealthcarestats.org) }\end{array}$ & $\begin{array}{l}\text { Universal health coverage } \\
\text { (\%) (healthdata.org) }\end{array}$ & $\begin{array}{l}\text { Classification by World Bank } \\
\text { (world bank data.org 2020) }\end{array}$ \\
\hline Nigeria & 2030 & 13 & 38.3 & Low-middle income \\
\hline Ethiopia & 850 & 28 & 46.5 & Low income \\
\hline Sudan & 590 & 19 & 51.8 & Low income \\
\hline Egypt & 2690 & 29 & 54.8 & Low-middle income \\
\hline DR Congo & 530 & 12 & 45.2 & Low income \\
\hline $\begin{array}{l}\text { Sierra } \\
\text { Leone }\end{array}$ & 540 & 11 & 42.1 & Low income \\
\hline Libya & 7640 & 63 & 66.3 & Upper-middle income \\
\hline Cameron & 1500 & 13 & 42.3 & Low-middle income \\
\hline Senegal & 1460 & 33 & 49.6 & Low-middle income \\
\hline Rwanda & 830 & 34 & 59.4 & Low income \\
\hline $\begin{array}{l}\text { South } \\
\text { Africa }\end{array}$ & 6040 & 54 & 59.7 & Upper-middle income \\
\hline Kenya & 1750 & 36 & 51.6 & Low-middle income \\
\hline $\begin{array}{l}\text { Cote } \\
\text { d'lvoire }\end{array}$ & 2290 & 26 & 43.0 & Low-middle income \\
\hline Tanzania & 1080 & 41 & 55.2 & Low-middle income \\
\hline Uganda & 780 & 17 & 52.7 & Low income \\
\hline Morocco & 3190 & 47 & 58.0 & Low-middle income \\
\hline
\end{tabular}

sample size, year of publication, and professions of HCWs.

The prevalence of hepatitis B was found to be highest in Western Africa 11.67\% (95\% CI 8.21-15.17), and the lowest was reported from Northern Africa $3.50 \%$ (95\% CI 2.41-4.58). Heterogeneity has been shown to vary from $\mathrm{I}^{2}=72$ to $91.6 \%$ on this estimate (Table 4 ). This meta-analysis also found that the prevalence of hepatitis $\mathrm{C}$ infection varied between different sub-regions of Africa, and the highest prevalence was found in Northern Africa, 11.23\% (95\% CI 5.45-17.01), with significant heterogeneity $\mathrm{I}^{2}=97.8 \%$ and the lowest prevalence was identified in Eastern Africa 1.32\% (95\% CI 0.16-2.47) (Table 5).

The analysis of sub-group by sample size identified that highest prevalence of HBV among studies with sample size < 101, 11.33\% (95\% CI 6.17-16.50), and lowest prevalence was identified among sample size between 101 and 384, 5.57 (95\% CI 4.31-6.83) (Table 4). Similarly, the highest prevalence of HCV among studies with sample size < 101, 14.28\% (95\% CI 1.16-27.40) and lowest prevalence was identified among sample size between 101 and 384, 1.19 (95\% CI 0.54-1.84) (Table 5). The heterogeneity was shown to vary from 76.0 to $97.6 \%$ for HBV and from 21.8 to $97.6 \%$ for HCV.

In the sub-group analysis of studies by year of publication, highest prevalence of HBV was revealed by studies conducted before 2001, 12.33 (95\% CI 3.32-21.39), and lowest among studies conducted between 2011 and
2021, 5.71 (95\% CI 4.68-6.74) (Table 4). For HCV, lowest prevalence was revealed by studies conducted before 2001, 3.74 (95\% CI 2.24-5.23), and highest among studies conducted between 2011 and 2021, 17.94 (95\% CI 17.94-24.75) (Table 5).

The sub-group was also conducted by profession of HCWs for HBV, in which laboratory staffs were identified to have relatively highest prevalence, 7.32 (95\% CI 3.77-10.88), and lowest prevalence were identified among physician 6.30 (95\% CI 3.54-9.07). The heterogeneity was shown to vary from $59.7 \%$ among laboratory staff and to $84.4 \%$ among nurses (Table 4 ).

\section{Sensitivity analysis}

To detect the source of heterogeneity, a leave-one-out sensitivity analysis was employed. The result of sensitivity analysis using the random-effects model revealed that there was no single study that influenced the overall prevalence of hepatitis $\mathrm{B}$ and $\mathrm{C}$ infection among HCWs (Supplementary file 3).

\section{The publication biases}

The presence of publication bias was evaluated using funnel plots and Begg's tests at a significance level of less than 0.05 . The findings revealed that publication bias was significant for the studies on the prevalence of hepatitis B $(p<0.001)$ (Fig. 4). Similarly, it was statistically significant $(p=0.001)$ for the prevalence of hepatitis $C$ among health care workers. The trim and fill analysis 


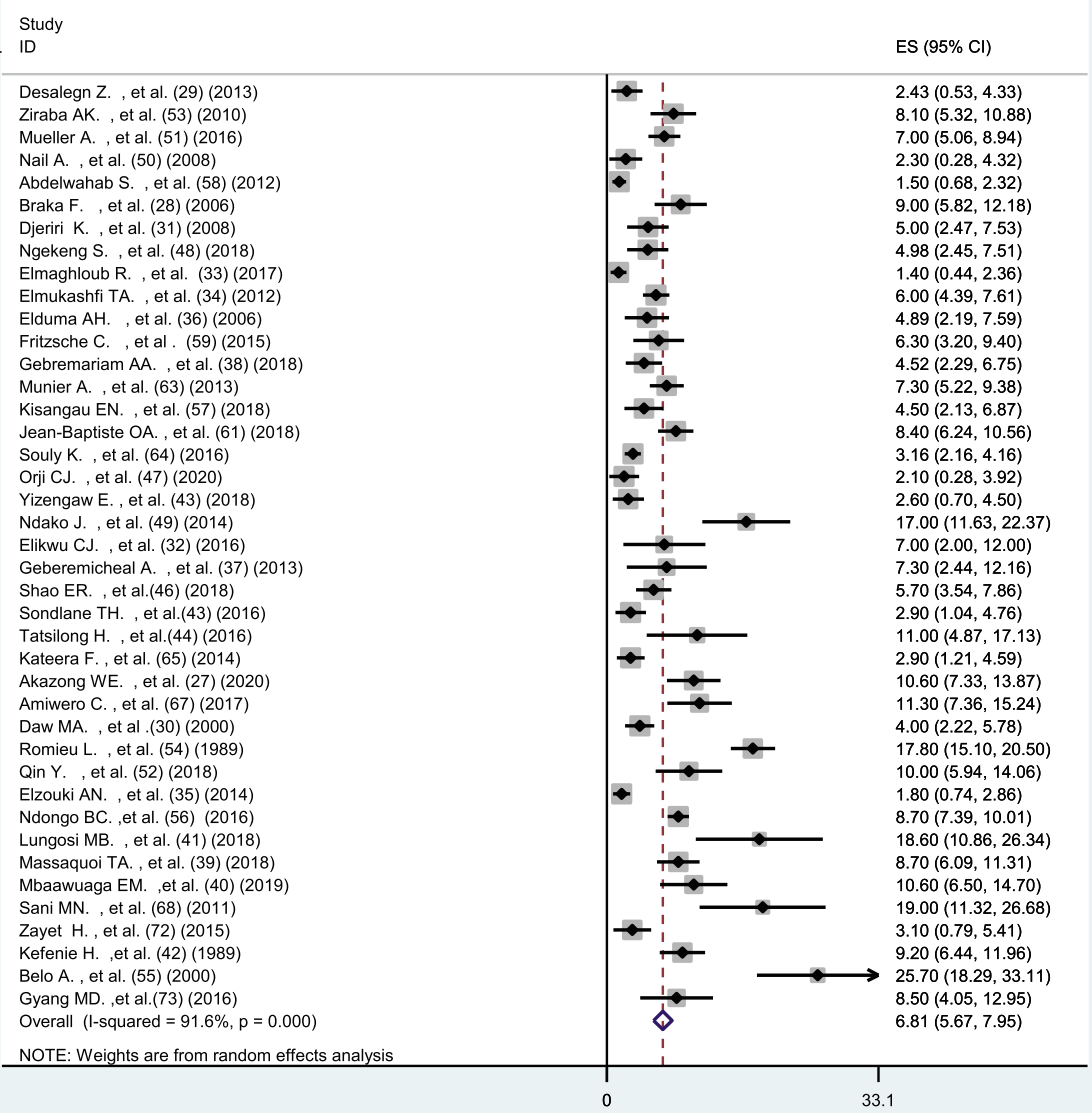

Fig. 2 Forest plot showing the pooled prevalence of hepatitis B among HCWs in Africa

added twenty-nine studies and the pooled prevalence of hepatitis B in Africa varied to 9.1\% (95\% CI 7.1-11.7), while nine studies were added, and pooled prevalence of hepatitis C in Africa varied to 1.32 (95\% CI 0.92-1.55).

\section{Meta-regression}

In a meta-regression analysis, the publication year and sample size were not significant sources of heterogeneity for the prevalence of hepatitis B. In this study, no significant relationship was identified between the prevalence of hepatitis B and the publication year $(p$-value $=0.35)$ and sample size $(p$-value $=0.46)$. Likewise, there was no significant association between the prevalence of hepatitis $\mathrm{C}$ and the publication year $(p$-value $=0.67)$ and sample size $(p$-value $=0.84)$.

\section{Discussion}

This is the first review and meta-analysis conducted in Africa on the prevalence of hepatitis $B$ and $C$ among HCWs. The pooled prevalence of hepatitis B and C among HCWs in Africa was $6.81 \%$ and $5.58 \%$, respectively. The highest and lowest prevalence of HBV was identified in the western and northern parts of Africa, respectively.

The pooled prevalence of HBV among HCWs in Africa was not shown to have a significant difference from the general population (6.1\%) [75]. The current finding was almost similar to a review conducted in Thailand which has revealed the pooled prevalence of hepatitis B among HCWs to be 5.2\% [76]. The present prevalence of HBV identified by our analysis is higher than a review conducted in 2017 in Middle Eastern countries [77] and a study conducted in Turkey which has revealed hepatitis B prevalence among HCWs to be $3 \%$ [78]. It was also found to be much higher than the pooled prevalence of hepatitis B among $\mathrm{HCW}$ s reported in Iranians (0.4\%) [79] and Brazilian 0.8\% [80]. This may be due to the higher vaccination status of Iranians against hepatitis B [81] and the low vaccination status of African HCWs against hepatitis B [23]. Further, in developing countries, more than half of HBV infections in HCWs were attributable to percutaneous occupational exposure [21, 82]. 


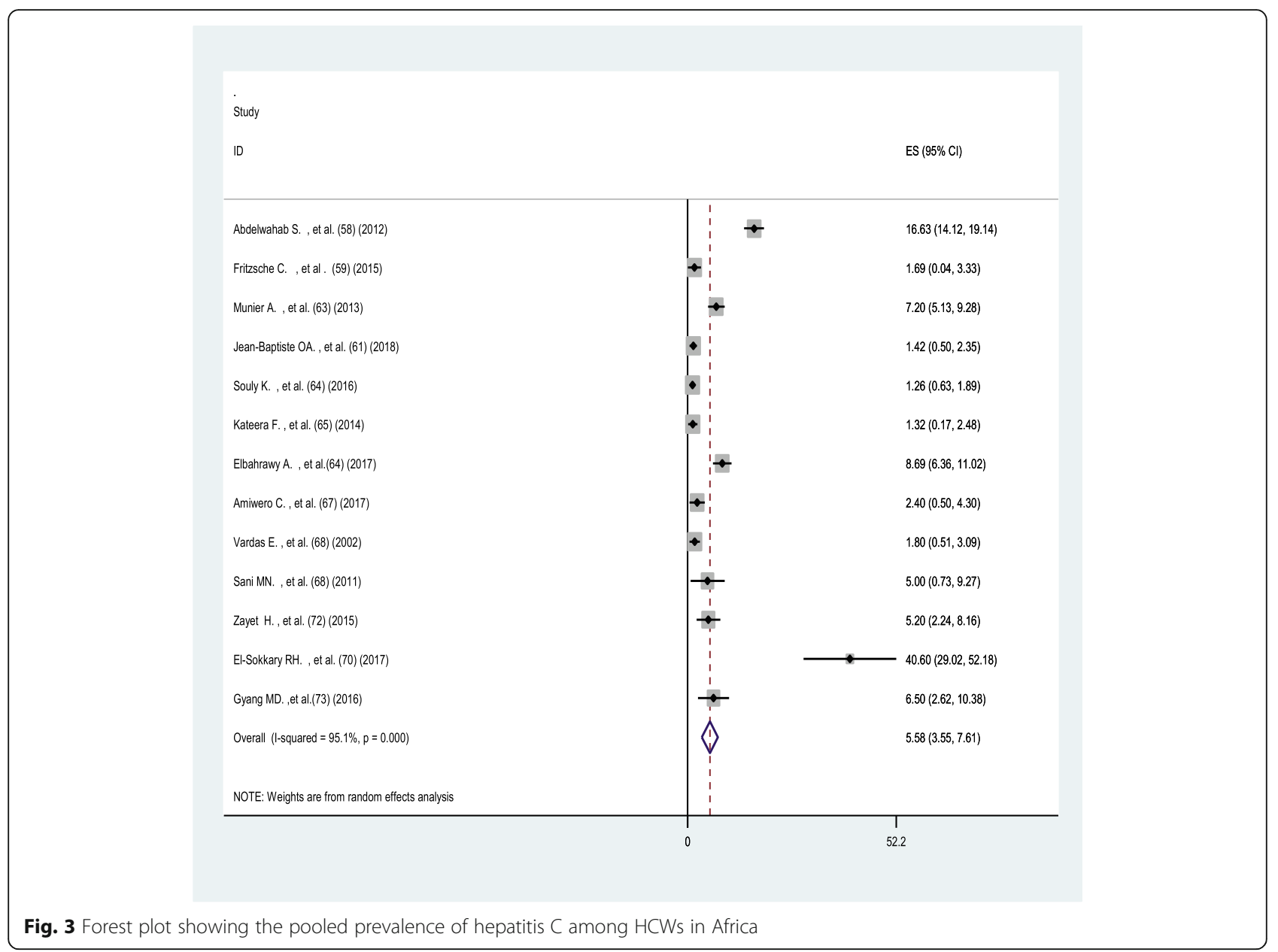

The current review has revealed the prevalence of $\mathrm{HCV}$ among HCWs in Africa was nearly two times higher when compared with the prevalence in the general population $(2.9 \%)$ [83]. This difference may be due to HCWs vulnerability towards occupational blood and body flood exposure than the general population. This finding is significantly higher when compared with the pooled prevalence reported in Germany (0.04\%) [84], Turkey (0.3\%) [78], and Scotland (0.28\%) [85]. The possible reason for the discrepancy may be due to higher frequencies of healthcare associated infection in Africa than developed countries [86].

In this study, we found a variation in hepatitis B prevalence among HCWs across African regions. The subgroup analysis has revealed that the highest and lowest prevalence of hepatitis B among HCWs was found in the western and northern parts of Africa, respectively. The difference can be explained by the vaccination status of HCWs in the region. In the northern part of Africa, about $62 \%$ of HCWs have fully vaccinated against hepatitis B [23]. While only $30 \%$ of HCWs have been fully vaccinated in the western part of Africa [23]. The highest prevalence of $\mathrm{HCV}$ was identified in the northern part of Africa. The difference might be due to the higher occupational exposure rate of blood and body fluid in the northern part of Africa [21].

The strengths of this review and meta-analysis were comprehensive search through all reachable databases and rigorously following the PRISMA statement in all processes of conducting this systematic and meta-analysis.

This meta-analysis was reported with the following limitations. First, some studies included in this review had small sample sizes $(n<100 \mathrm{HCWs})$ which may affect the estimated prevalence of HBV and HCV in HCWs. As revealed by the sub-group analysis studies with a small sample size have shown higher prevalence of HBV (Table 4) and HCV (Table 5) among HCWs in Africa. This is supported by the fact that a small sample size will have a wider confidence interval and overestimate the magnitude of the association [87]. In addition, studies with a wide confidence interval are related to the low precision of the finding [88]. Second, there is a significant publication bias of included studies. Publication bias is bias caused by unpublished studies and usually researches with negative results less likely to be published [89]. Unpublished articles are not easily available in areas where there are no repositories. This is the most common problem in Africa 
Table 4 Showing sub-group analysis of HBV prevalence by sample size, year of publication, regions of Africa, and HCWs professions in Africa

\begin{tabular}{|c|c|c|c|c|}
\hline & Prevalence of $\mathrm{HBsAg}$ & 95\% Confidence interval & Heterogeneity $\left(I^{2} \%\right)$ & $P$-value \\
\hline \multicolumn{5}{|c|}{ Sub-group analysis by sample size } \\
\hline 1. $<101$ & 11.33 & $6.17-16.50$ & 76.0 & $P=0.002$ \\
\hline 2. $101-384$ & 5.57 & $4.31-6.83$ & 85.7 & $P<0.001$ \\
\hline 3. $385-1000$ & 6.42 & $4.24-8.61$ & 95.1 & $P<0.001$ \\
\hline 4. $>1000$ & 5.91 & $0.48-11.54$ & 97.6 & $P<0.001$ \\
\hline \multicolumn{5}{|c|}{ Sub-group analysis by year of publication } \\
\hline 1. $<2001$ & 12.32 & $3.32-21.39$ & 97.1 & $P<0.001$ \\
\hline 2. $2001-2010$ & 5.79 & $3.43-8.16$ & 88.3 & $P<0.001$ \\
\hline 3. $2011-2021$ & 5.71 & $4.68-6.74$ & 87.7 & $P<0.001$ \\
\hline \multicolumn{5}{|c|}{ Sub-group analysis by regions of Africa } \\
\hline 1. North Africa & 3.50 & $2.41-4.58$ & 83.8 & $P<0.001$ \\
\hline 2. East Africa & 5.51 & $4.03-6.99$ & 77.5 & $P<0.001$ \\
\hline 3. Middle Africa & 8.77 & $6.32-11.21$ & 72.2 & $P=0.003$ \\
\hline 4. Western Africa & 11.69 & $8.21-15.17$ & 91.8 & $P<0.001$ \\
\hline 5. Southern Africa & 2.90 & $1.04-4.99$ & - & - \\
\hline \multicolumn{5}{|c|}{ Sub-group analysis by professions } \\
\hline 1. Physician & 6.30 & $3.54-9.07$ & 81.8 & $P<0.001$ \\
\hline 2. Nurses & 6.31 & $4.23-8.40$ & 84.5 & $P<0.001$ \\
\hline 3. Laboratory staff & 7.32 & $3.77-10.88$ & 59.4 & $P=0.003$ \\
\hline
\end{tabular}

since most research institutes and universities do not have repositories available online [90].

\section{Conclusion}

The prevalence of hepatitis $\mathrm{B}$ is more than one in fifteen HCWs in Africa. While about one in twenty HCWs are affected by the hepatitis $C$ virus. This high prevalence shows that hepatitis $\mathrm{B}$ and $\mathrm{C}$ are still endemic among HCWs in Africa. To reduce the prevalence of $\mathrm{HCV}$ and $\mathrm{HBV}$ among HCWs it needs a new strategy that reduces occupational exposure to blood and body fluids. Including mandatory vaccination against hepatitis $B$ is required for HCWs as they are among the risky groups in the community

Table 5 Showing sub-group analysis of HCV prevalence by sample size, year of publication, and sub-regions of African countries

\begin{tabular}{|c|c|c|c|c|}
\hline & Prevalence of HCV & 95\% confidence interval & Heterogeneity $\left(I^{2} \%\right)$ & $P$-value \\
\hline \multicolumn{5}{|c|}{ Sub-group analysis by sample size } \\
\hline 1. $<101$ & 14.28 & $1.16-27.40$ & 94.8 & $P=0.001$ \\
\hline 2. $101-384$ & 1.19 & $0.54-1.84$ & 21.8 & $P=0.269$ \\
\hline 3. 385-1000 & 7.04 & $2.46-11.62$ & 97.6 & $P<0.001$ \\
\hline 4. $>1000$ & 1.26 & $0.67-1.85$ & - & - \\
\hline \multicolumn{5}{|c|}{ Sub-group analysis by year of publication } \\
\hline 1. $<2011$ & 3.74 & $2.24-5.23$ & 94.2 & $P<0.001$ \\
\hline 2. $2011-2021$ & 17.94 & $17.94-24.75$ & 95.7 & $P<0.001$ \\
\hline \multicolumn{5}{|c|}{ Sub-group analysis by regions of Africa } \\
\hline 1. North Africa & 11.23 & $5.76-17.02$ & 97.8 & $P<0.001$ \\
\hline 2. East Africa & 1.32 & $0.17-2.48$ & - & - \\
\hline 3. Middle Africa & 1.69 & $0.004-3.33$ & - & - \\
\hline 4. Western Africa & 3.04 & $1.08-4.99$ & 65.5 & $P<0.033$ \\
\hline 5. South Africa & 2.90 & $1.04-4.78$ & - & - \\
\hline
\end{tabular}




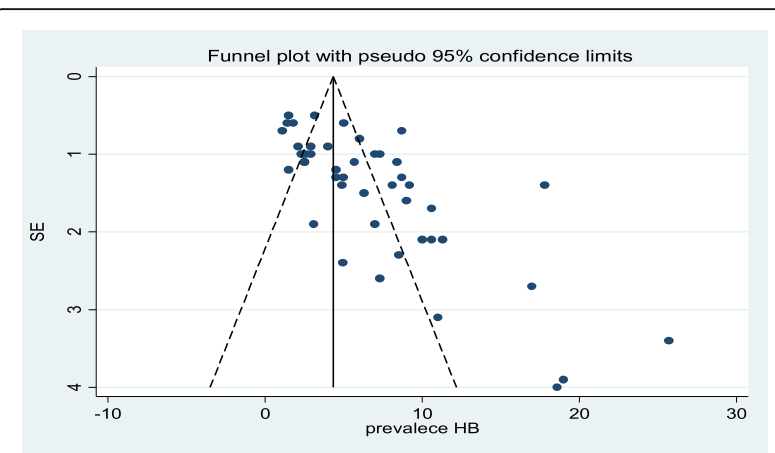

Fig. 4 Funnel plot showing publication bias of studies included for the prevalence of hepatitis B among health care workers in Africa

and reduction of occupational exposure by maintaining adequate personal protective equipment supported by regulations. In addition, continuous training on infection prevention procedures for all HCWs should be provided.

\section{Supplementary Information}

The online version contains supplementary material available at https:/doi. org/10.1186/s12199-021-00983-9.

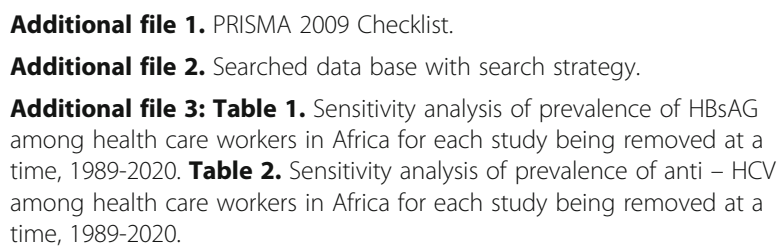

\section{Acknowledgements}

We sincerely thank all the authors of original articles who have responded timely to our queries through emails.

\section{Authors' contributions}

DA: Conceptualize, study protocol, data extraction and analysis, and writing the original draft of the manuscript. DA, BS, and ZT conducted study design, literature review, and statistical analysis of the review. DA, BS, and ZT conducted a critical appraisal and data extraction. DA wrote the original draft of the manuscript. $\mathrm{ZT}, \mathrm{BS}$, and DA critically revised the manuscript. The authors read and approved the final manuscript.

\section{Funding}

Nil

\section{Availability of data and materials}

The part of the data analyzed during this study is included in this manuscript. Other data will be available from the corresponding author upon a reasonable request.

\section{Declarations}

Ethics approval and consent to participate

Not applicable

\section{Consent for publication}

Not applicable

\section{Competing interests}

The authors declare that they have no competing interests.

\section{Author details}

'Department of Human Anatomy, School of Medicine, Goba Referral Hospital, Madda Walabu University, Goba, Ethiopia. ${ }^{2}$ Department of Public Health, School of Health Science, Goba Referral Hospital, Madda Walabu University, Goba, Ethiopia. ${ }^{3}$ Department of Public Health, College of Medicine and Health Sciences, Dire Dawa University, Dire Dawa, Ethiopia.

Received: 3 March 2021 Accepted: 23 May 2021

Published online: 02 June 2021

\section{References}

1. Te HS, Jensen DM. Epidemiology of hepatitis B and C viruses: a global overview. Clin Liver Dis. 2010;14(1):1-21. https://doi.org/10.1016/j.cld.2 009.11.009.

2. Muvunyi CM, Harelimana JDD, Sebatunzi OR, Atmaprakash AC, Seruyange E, Masaisa F, et al. Hepatitis B vaccination coverage among healthcare workers at a tertiary hospital in Rwanda. BMC Res Notes. 2018;11(1):1-5. https://doi. org/10.1186/s13104-018-4002-5.

3. Budak GG, Gülenç N, Özkan E, Bülbül R, et al. Seroprevalences of hepatitis B and hepatitis $C$ among healthcare workers in Tire State Hospital. Dicle Med J. 2017:44(3):267-70.

4. Westermann C, Peters C, Lisiak B, Lamberti M, Nienhaus A. The prevalence of hepatitis $C$ among healthcare workers: a systematic review and metaanalysis. Occup Environ Med. 2015;72(12):880-8. https://doi.org/10.1136/ oemed-2015-102879.

5. Alam S, Ahmad N, Khan M, Mustafa G, Al Mamun A, Mashud G. Hepatitis C virus infection among health care workers. J Bangladesh Coll Physicians Surg. 2007;25(3):126-9.

6. Ogoina D, Pondei K, Adetunji B, Chima G, Isichei C, Gidado S. Prevalence of hepatitis B vaccination among health care workers in Nigeria in 2011-12. Int J Occup Environ Med. 2014;5(1):51-6.

7. Westermann C, Dulon M, Wendeler D, Nienhaus A. Hepatitis C among healthcare personnel: secondary data analyses of costs and trends for hepatitis C infections with occupational causes. J Occup Med Toxicol. 2016; 11(1):1-8. https://doi.org/10.1186/s12995-016-0142-5.

8. Hatzakis A, Wait S, Bruix J, Buti M, Carballo M, Cavaleri M, et al. The state of hepatitis $B$ and $C$ in Europe: report from the hepatitis $B$ and $C$ summit conference. J Viral Hepat. 2011;18(1):1-16. https://doi.org/10.1111/j.1365-2 893.2011.01499.x.

9. Ishizaki A, Bouscaillou J, Luhmann N, Liu S, Chua R, Walsh N, et al. Survey of programmatic experiences and challenges in delivery of hepatitis $B$ and $C$ testing in low- and middle-income countries. BMC Infect Dis. 2017;17(1): 130-40.

10. Aaron D, Nagu TJ, Rwegasha J, Komba E. Hepatitis B vaccination coverage among healthcare workers at national hospital in Tanzania: how much, who and why? BMC Infect Dis. 2017;17(1):1-7.

11. Abebaw TA, Aderaw Z, Gebremichael B. Hepatitis B virus vaccination status and associated factors among health care workers in Shashemene Zonal Town, Shashemene, Ethiopia: a cross sectional study. BMC Res Notes. 2017; 10(1):1-9.

12. Hadler SC. Hepatitis B virus infection and health care workers. Vaccine. 1990; $8(1): 24-8$.

13. Ssekamatte T, Mukama T, Kibira SPS, Ndejjo R, Bukenya JN, Kimoga ZPA, et al. Hepatitis B screening and vaccination status of healthcare providers in Wakiso district, Uganda. PLoS One. 2020;15(7):1-13.

14. Abeje G, Azage M. Hepatitis B vaccine knowledge and vaccination status among health care workers of Bahir Dar City Administration, Northwest Ethiopia: a cross sectional study. BMC Infect Dis. 2015;15(1):1-6.

15. Nitin DS, Prashant, Nitin A, Prashant P, Kumar DH. Status of protection against hepatitis B infection among healthcare workers ( $\mathrm{HCW}$ ) in a tertiary healthcare center in India: results can't be ignored! J Hematol Clin Res. 2017:2(1):001-5.

16. Adenlewo OJ, Adeosun PO, Fatusi OA. Medical and dental students' attitude and practice of prevention strategies against hepatitis B virus infection in a Nigerian university. Pan Afr Med J. 2017;28(33):1-8.

17. Ziglam H, El-Hattab M, Shingheer N, Zorgani A, Elahmer O. Hepatitis B vaccination status among healthcare workers in a tertiary care hospital in 
Tripoli, Libya. J Infect Public Health. 2013;6(4):246-51. https://doi.org/10.101 6/j.jiph.2013.02.001.

18. Kisic-Tepavcevic D, Kanazir M, Gazibara T, Maric G, Makismovic N, Loncarevic $\mathrm{G}$, et al. Predictors of hepatitis $B$ vaccination status in healthcare workers in Belgrade, Serbia, December 2015. Eurosurveillance. 2017;22(16):0-8. https:// doi.org/10.2807/1560-7917.ES.2017.22.16.30515.

19. Thomas RJ, Fletcher GJ, Kirupakaran H, Chacko MP, Thenmozhi S, Eapen CE, et al. Prevalence of non-responsiveness to an indigenous recombinant hepatitis B vaccine: a study among South Indian health care workers in a tertiary hospital. Indian J Med Microbiol. 2015;33(1):S32-6. https://doi.org/1 0.4103/0255-0857.150877.

20. Khan FY, Ross AJ. Hepatitis B immunisation amongst doctors and laboratory personnel in Kwazulu-natal, South Africa. Afr J Prim Heal Care Fam Med. 2013:5(1):1-6.

21. Auta A, Adewuyi EO, Tor-Anyiin A, Aziz D, Ogbole E, Ogbonna BO, et al. Health-care workers' occupational exposures to body fluids in 21 countries in Africa: systematic review and meta-analysis. Bull World Health Organ. 2017;95(12):831-41. https://doi.org/10.2471/BLT.17.195735

22. Sahiledengle B, Tekalegn Y, Woldeyohannes D, Quisido BJE. Occupational exposures to blood and body fluids among healthcare workers in Ethiopia: a systematic review and meta-Analysis. Environ Health Prev Med. 2020;25(1): $1-14$.

23. Auta A, Adewuyi EO, Kureh GT, Onoviran N, Adeloye D. Hepatitis B vaccination coverage among health-care workers in Africa: a systematic review and meta-analysis. Vaccine. 2018;36(32):4851-60. https://doi.org/10.1 016/j.vaccine.2018.06.043.

24. Perz JF, Armstrong GL, Farrington LA, Hutin YJF, Bell BP. The contributions of hepatitis $B$ virus and hepatitis $C$ virus infections to cirrhosis and primary liver cancer worldwide. J Hepatol. 2006;45(4):529-38. https://doi.org/10.101 6/j.jhep.2006.05.013.

25. Abiola AHO, Agunbiade AB, Badmos KB, Lesi AO, Lawal AO, Alli QO. Prevalence of $\mathrm{HBsAg}$, knowledge, and vaccination practice against viral hepatitis $b$ infection among doctors and nurses in a secondary health care facility in Lagos state, south-western Nigeria. Pan Afr Med J. 2016:23:1-10.

26. Alese $\mathrm{OO}$, Alese MO, Ohunakin A, Oluyide PO. Seroprevalence of hepatitis B surface antigen and occupational risk factors among health care workers in Ekiti state, Nigeria. J Clin Diagn Res. 2016;10(2):LC16-8.

27. Akazong WE, Tume C, Njouom R, Ayong L, Fondoh V, Kuiate JR. Knowledge, attitude and prevalence of hepatitis $B$ virus among healthcare workers: a cross-sectional, hospital-based study in Bamenda Health District, NWR, Cameroon. BMJ Open. 2020;10(3):1-8.

28. Braka F, Nanyunja M, Makumbi I, Mbabazi W, Kasasa S, Lewis RF. Hepatitis B infection among health workers in Uganda: evidence of the need for health worker protection. Vaccine. 2006;24(47-48):6930-7. https://doi.org/10.1016/j. vaccine.2006.08.029

29. Desalegn Z, Selassie SG. Prevalence of hepatitis B surface antigen (HBsAg) among health professionals in public hospitals in Addis Ababa, Ethiopia. Ethiop J Heal Dev. 2013;27(1):72-9.

30. Daw MA, Siala IM, Warfalli MM, Muftah MI. Seroepidemiology of hepatitis B virus markers among hospital health care workers: analysis of certain potential risk factors. Saudi Med J. 2000;21(12):1157-60.

31. Djeriri K, Laurichesse H, Merle J I, Charof R, Abouyoub A, Fontana L, et al. Hepatitis B in Moroccan health care workers. Occup Med (Chic III). 2008; 58(6):419-24.

32. Elikwu CJ, Shonekan O, Shobowale E, Nwadike V, Tayo B, Okangba C, et al. Seroprevalence of hepatitis B surface antigenaemia among healthcare worker in a private Nigerian tertiary health institution. Int J Infect Control. 2016;12(2):1-6,

33. Elmaghloub R, Elbahrawy A, El Didamony G, Elwassief A, Saied Mohammad A-G, Alashker A, et al. Hepatitis B virus genotype E infection among Egyptian health care workers. J Transl Intern Med. 2017;5(2):100-5. https:// doi.org/10.1515/jtim-2017-0012.

34. Elmukashfi $T A$, Elkhidir IM, Ibrahim OA, Bashir AA, Elkarim MAA. Hepatitis $B$ virus infection among health care workers in public teaching hospitals in Khartoum State, Sudan. Saf Sci. 2012;50(5):1215-7. https://doi.org/10.1016/j. ssci.2011.12.028.

35. Elzouki AN, Elgamay SM, Zorgani A, Elahmer O. Hepatitis B and C status among health care workers in the five main hospitals in eastern Libya. J Infect Public Health. 2014;7(6):534-41. https://doi.org/10.1016/j.jiph.2014.07.006.

36. Elduma AH, Saeed NS. Hepatitis B virus infection among staff in three hospitals in Khartoum, Sudan, 2006-07. East Mediterr Health J. 2011;17(6): 2011.
37. Geberemicheal A, Gelaw A, Moges F, Dagnaw M. Seroprevalence of hepatitis B virus infections among health care workers at the Bulle Hora Woreda Governmental Health Institutions, Southern Oromia, Ethiopia. J Environ Occup Sci. 2013;2(1):9. https://doi.org/10.5455/jeos.20130220105759.

38. Gebremariam AA, Tsegaye AT, Shiferaw YF, Reta M, Getaneh A. Seroprevalence of hepatitis B virus infection and associated factors among mothers in Gondar, North-west Ethiopia: a population based study. Ethiop Med J. 2019:57(2):97-106.

39. Massaquoi TA, Burke RM, Yang G, Lakoh S, Sevalie S, Li B, et al. Cross sectional study of chronic hepatitis B prevalence among healthcare workers in an urban setting, Sierra Leone. PLoS One. 2018;13(8):1-12.

40. Mbaawuaga EM, Hembah-hilekaan SK, Iroegbu CU, Ike AC. Hepatitis B virus and human immunodeficiency virus infections among health care workers in some health care centers in Benue State, Nigeria; 2019. p. 48-62. Available from: https://www.scirp.org/pdf/OJMM_2019061815320787.pdf

41. Lungosi MB, Muzembo BA, Mbendi NC, Nkodila NA, Ngatu NR, Suzuki T, et al. Assessing the prevalence of hepatitis B virus infection among health care workers in a referral hospital in Kisantu, Congo DR: A pilot study. Ind Health. 2019:57(5):621-6. https://doi.org/10.2486/indhealth.2018-0166.

42. Kefenie H, Desta B, Abebe A. Prevalence of hepatitis B infection among hospital personnel in Addis Ababa (Ethiopia). Environ Prot. 1993;5(4):489-96.

43. Yizengaw E, Getahun T, Geta M, Mulu W, Ashagrie M, Hailu D, et al. Seroprevalence of hepatitis $B$ virus infection and associated factors among health care workers and medical waste handlers in primary hospitals of North-west Ethiopia. BMC Res Notes. 2018;11(1):4-9.

44. Tatsilong HOP, Noubiap JJN, Nansseu JRN, Aminde LN, Bigna JJR, Ndze VN, et al. Hepatitis B infection awareness, vaccine perceptions and uptake, and serological profile of a group of health care workers in Yaoundé, Cameroon. BMC Public Health. 2016;16(1):1-7.

45. Sondlane TH, Mawela L, Razwiedani LL, Selabe SG, Lebelo RL, Rakgole JN, et al. High prevalence of active and occult hepatitis $B$ virus infections in healthcare workers from two provinces of South Africa. Vaccine. 2016;34(33): 3835-9. https://doi.org/10.1016/j.vaccine.2016.05.040.

46. Shao ER, Mboya IB, Gunda DW, Ruhangisa FG, Temu EM, Nkwama ML, et al. Seroprevalence of hepatitis B virus infection and associated factors among healthcare workers in northern Tanzania. BMC Infect Dis. 2018;18(1):1-10.

47. Orji CJ, Chime OH, Ndibuagu EO. Vaccination status and prevalence of hepatitis $B$ virus infection among health-care workers in a tertiary health institution, Enugu State, Nigeria. Proc Singapore Healthc. 2020;29(2):119-25. https://doi.org/10.1177/2010105820923681.

48. Ngekeng S, Chichom-Mefire A, Nde P, Nsagha D, Nkuigue A, Tiogouo K, et al. Hepatitis B prevalence, knowledge and occupational factors among health care workers in Fako Division, South West Region Cameroon. Microbiol Res J Int. 2018;23(4):1-9. https://doi.org/10.9734/MRJI/2018/40445.

49. Ndako J, Onwuliri E, Adelani-Akande T, Olaolu D, Dahunsi S, Udo U. Screening for hepatitis B surface antigen ( $\mathrm{HBsAg}$ ) among health care workers (HCW) in an urban community South-South Nigeria. Int J Biol Pharm Allied Sci. 2014;3(3):415-25.

50. Nail A, Eltiganni S, Imam A. Seroprevalence of hepatitis B and C among health care workers in Omdurman, Sudan. Sudan JMS. 2008;3(3):201-5.

51. Mueller A, Stoetter L, Kalluvya S, Stich A, Majinge C, Weissbrich B, et al. Prevalence of hepatitis $B$ virus infection among health care workers in a tertiary hospital in Tanzania. BMC Infect Dis. 2015;15(1):1-9. https://doi.org/1 0.1186/s12879-015-1129-z.

52. Qin YL, Li B, Zhou YS, Zhang X, Li L, Song B, et al. Prevalence and associated knowledge of hepatitis $B$ infection among healthcare workers in Freetown, Sierra Leone. BMC Infect Dis. 2018;18(1):1-8.

53. Ziraba AK, Bwogi J, Namale A, Wainaina CW, Mayanja-Kizza H. Seroprevalence and risk factors for hepatitis B virus infection among health care workers in a tertiary hospital in Uganda. BMC Infect Dis. 2010;10:191.

54. Romieu I, Sow I, Lu S, Laroque G, Prince-David M, Romet-Lemonne JL. Prevalence of hepatitis B markers among hospital workers in Senegal. J Med Virol. 1989;27(4):282-7. https://doi.org/10.1002/jmv.1890270405.

55. Belo A. Prevalence of hepatitis b virus markers in surgeons in lagos, nigeria. East Afr Med J. 2000;77(5):283-5. https://doi.org/10.4314/eamj.v77i5.46634.

56. Ndongo BC, Eteki L, Siedner M, Mbaye R, Chen J, Ntone R, et al. Prevalence and vaccination coverage of Hepatitis $B$ among healthcare workers in Cameroon: a national seroprevalence survey. J Viral Hepat. 2018;25(12): 1582-7. https://doi.org/10.1111/jvh.12974.

57. Kisangau EN, Awour A, Juma B, Odhiambo D, Muasya T, Kiio SN, et al. Prevalence of hepatitis $B$ virus infection and uptake of hepatitis $B$ vaccine 
among healthcare workers, Makueni County, Kenya 2017. J Public Health (United Kingdom). 2019:41(4):765-71.

58. Abdelwahab S, Rewisha E, Hashem M, Sobhy M, Galal I, Allam WR, et al. Risk factors for hepatitis $C$ virus infection among Egyptian healthcare workers in a national liver diseases referral centre. Trans R Soc Trop Med Hyg. 2012; 106(2):98-103. https://doi.org/10.1016/j.trstmh.2011.10.003.

59. Fritzsche C, Becker F, Hemmer CJ, Riebold D, Klammt S, Hufert F, et al. Hepatitis B and C: neglected diseases among health care workers in Cameroon. Trans R Soc Trop Med Hyg. 2013;107(3):158-64. https://doi.org/1 0.1093/trstmh/trs087.

60. Hebo HJ, Gemeda DH, Abdusemed KA. Hepatitis B and C Viral Infection: Prevalence, knowledge, attitude, practice, and occupational exposure among healthcare workers of Jimma University Medical Center, Southwest Ethiopia. Sci World J. 2019:1-11.

61. Jean-Baptiste OA, Amadou K, Mamadou D, Fabrice A, Sroboua TA, N'guessan N. Predictive factors for viral B and C infection in health workers in a university hospital in Ivory Coast. Open J Gastroenterol. 2018;8(10):37785. https://doi.org/10.4236/ojgas.2018.810039.

62. Mafopa NG, Giovanetti M, Wadoum REG, Minutolo A, Yinda CK, Russo G, et al. Viral hepatitis $B$ and $C$ detection among Ebola survivors and health care workers in Makeni, Sierra Leone. J Biosci Med. 2020;8(10):18-32.

63. Munier A, Marzouk D, Abravanel F, El-Daly M, Taylor S, Mamdouh R, et al. Frequent transient hepatitis $C$ viremia without seroconversion among healthcare workers in Cairo, Egypt. PLoS One. 2013;8(2):1-9. https://doi.org/1 0.1371/journal.pone.0057835.

64. Souly K, El Kadi MA, Elkamouni Y, Biougnach H, Kreit S, Zouhdi M. Prevalence of hepatitis $B$ and $C$ virus in health care personnel in lbn Sina Hospital, Rabat, Morocco. Open J Med Microbiol. 2016;6(1):17-22. https:// doi.org/10.4236/ojmm.2016.61004

65. Kateera F, Walker TD, Mutesa L, Mutabazi V, Musabeyesu E, Mukabatsinda C, et al. Hepatitis $B$ and $C$ seroprevalence among health care workers in a tertiary hospital in Rwanda. Trans R Soc Trop Med Hyg. 2014;109(3):203-8.

66. Elbahrawy A, Elwassief A, Abdallah AM, Kasem A, Mostafa S, Makboul K, et al. Hepatitis $C$ virus exposure rate among health-care workers in rural Lower Egypt governorates. J Transl Intern Med. 2017;5(3):164-8. https://doi. org/10.1515/jtim-2017-0024.

67. Amiwero C, Nelson EA, Yusuf M, Olaosebikan OF, Adeboye MA, Adamu UG, et al. Knowledge, awareness and prevalence of viral hepatitis among health care workers (HCWs) of the Federal Medical Centre Bida, Nigeria. J Med Res. 2017:3(3):114-20

68. Vardas E, Ross MH, Sharp G, McAnerney J, Sim J. Viral hepatitis in South African healthcare workers at increased risk of occupational exposure to blood-borne viruses. J Hosp Infect. 2002;50(1):6-12. https://doi.org/10.1053/ jhin.2001.1143

69. Sani NM, Bitrus I, Sarki AM, Mujahid NS. Seroprevalence of Hepatitis B and C among healthcare workers in Dutse Metropolis Jigawa State, Nigeria. bioRxiv. 2018. https://doi.org/10.1101/327940.

70. El-Sokkary RH, Tash RME, Meawed TE, El Seifi OS, Mortada EM. Detection of hepatitis $\mathrm{C}$ virus (HCV) among health care providers in an Egyptian university hospital: different diagnostic modalities. Infect Drug Resist. 2017; 10:357-64. https://doi.org/10.2147/IDR.S145844.

71. Abuojokh A. Prevalence of hepatitis $B$ and $C$ viral infection among health care workers in Elmak Nimir University Hospital; 2016.

72. Zayet $H, A M$ EE-D, SM A, MR E-K. Hepatitis B and C virus infection among health care workers in General Surgery Department, Assiut Univers Ity Hospitals. Egypt J Occup Med. 2015;39(1):85-104.

73. Gyang MD, Madaki AJK, Dankyau M, Toma BO, Danjuma SA, Gyang BA. Prevalence and correlates of hepatitis $B$ and $C$ seropositivity among health care workers in a semi urban setting in Northern central Nigeria. Highland Med Res J. 2016;16(2):75-9.

74. Joanna Briggs Institute. Checklist for prevalence studies. Checkl Prevalance Stud. 2016;7 Available from: http://joannabriggs.org/assets/docs/critical-a ppraisal-tools/JBI_Critical_Appraisal-Checklist_for_Prevalence_Studies.pdf. Accessed 20 Nov 2020

75. Spearman CW, Afihene M, Ally R, Apica B, Awuku Y, Cunha L, et al. Hepatitis $B$ in sub-Saharan Africa: strategies to achieve the 2030 elimination targets. Lancet Gastroenterol Hepatol. 2017;2(12):900-9. https://doi.org/10.1016/S24 68-1253(17)30295-9.

76. Leroi C, Adam P, Khamduang W, Kawilapat S, Ngo-Giang-Huong N, Ongwandee $\mathrm{S}$, et al. Prevalence of chronic hepatitis B virus infection in
Thailand: a systematic review and meta-analysis. Int J Infect Dis. 2016;51:3643. https://doi.org/10.1016/j.jiji.2016.08.017.

77. Davoudi-Kiakalayeh A, Mohammadi R, Pourfathollah AA, Siery Z, DavoudiKiakalayeh S. Alloimmunization in thalassemia patients: new insight for healthcare. Int J Prev Med. 2019;10:144.

78. Ozsoy M, Oncul O, Cavuslu S, Erdemoglu A, And GE, Pahsa A. Seroprevalences of hepatitis B and C among health care workers in Turkey. J Viral Hepat. 2003;10(3):150-6. https://doi.org/10.1046/j.1365-2893.2003.004 04.x.

79. Sayehmiri K, Azami M, Borji M, Nikpay S, Chamani M. Seroprevalence of hepatitis B virus surface antigen ( $\mathrm{HBsAg}$ ) in Iranian health care workers: systematic review and meta-analysis study. J Occup Environ Heal. 2016;2(1): 47-57.

80. Malkappa SK, Sreekanth B. Hepatitis B and C in healthcare workers: prevalence, relation to vaccination and occupational factors. J Evol Med Dent Sci. 2014;3(15):3919-22. https://doi.org/10.14260/jemds/2014/2376.

81. Hiva S, Negar K, Mohammad-reza P, Gholam-reza G, Mohsen A. High level of vaccination and protection against hepatitis B with low rate of HCV infection markers among hospital health care personnel in north of Iran : a cross- sectional study. BMC Public Health. 2020;20(920):1-9.

82. Singhal V, Bora D, Singh $S$. Hepatitis B in health care workers: Indian scenario. J Lab Physicians. 2009:1(2):041-8.

83. Petruzziello A, Marigliano S, Loquercio G, Cozzolino A, Cacciapuoti C, Petruzziello A, et al. Global epidemiology of hepatitis C virus infection : an up-date of the distribution and circulation of hepatitis $C$ virus genotypes. World J Gastroenterol. 2016;22(34):7824-40. https://doi.org/10.3748/wjg.v22. i34.7824.

84. Sperle I, Steffen G, Leendertz SA, Sarma N, Beermann S, Thamm R, et al. Prevalence of hepatitis B, C, and D in Germany: results from a scoping review. Front Public Health. 2020:8:424.

85. Thorburn D, Dundas D, McCruden EAB, Cameron SO, Goldberg DJ, Symington IS, et al. A study of hepatitis $C$ prevalence in healthcare workers in the West of Scotland. Gut. 2001;48(1):116-20. https://doi.org/10.1136/ gut.48.1.116.

86. Nejad SB, Allegranzi B, Syed SB, Ellisc B, Pittetd D. Infections liées aux soins de santé en afrique: une étude systématique. Bull World Health Organ. 2011:89(10):757-65. https://doi.org/10.2471/BLT.11.088179.

87. Hackshaw A. Small studies: strengths and limitations. Eur Respir J. 2008;32(5): 1141-3. https://doi.org/10.1183/09031936.00136408.

88. Hirpara N, Jain S, Gupta A, Dubey S. Interpreting research findings with confidence interval. J Orthod Endod. 2015;1 (1):8 Available from: http:// orthodontics-endodontics.imedpub.com/interpreting-research-findings-withconfidence-interval.php?aid=7224.

89. Gillbody SM, Song F, Eastwood AJ, Sutton A. The causes, consequences and detection of publication bias in psychiatry. Acta Psychiatr Scand. 2000; 102(4):241-9. https://doi.org/10.1034/j.1600-0447.2000.102004241.x.

90. Ezema IJ. Local contents and the development of open access institutional repositories in Nigeria University libraries: challenges, strategies and scholarly implications. Libr Hi Tech. 2013;31(2):323-40. https://doi.org/10.11 08/07378831311329086.

\section{Publisher's Note}

Springer Nature remains neutral with regard to jurisdictional claims in published maps and institutional affiliations.

Ready to submit your research? Choose BMC and benefit from:

- fast, convenient online submission

- thorough peer review by experienced researchers in your field

- rapid publication on acceptance

- support for research data, including large and complex data types

- gold Open Access which fosters wider collaboration and increased citations

- maximum visibility for your research: over $100 \mathrm{M}$ website views per year

At $\mathrm{BMC}$, research is always in progress.

Learn more biomedcentral.com/submissions 